\title{
METODOLOGÍAS PARTICIPATIVAS PARA LA MEJORA DEL APRENDIZAJE EN EDUCACIÓN SUPERIOR. UN PROYECTO INNOVADOR CON ESTUDIANTES DE LA FACULTAD DE EDUCACIÓN
}

\begin{abstract}
Participating Methodologies for the Improvement of Higher Education Learning. An innovative project with students of the Faculty of Education
\end{abstract}

\author{
Natalia González Fernández \\ Universidad de Cantabria \\ gonzalen@unican.es \\ Jose Luis García González \\ Universidad de Cantabria \\ joseluis.garciag@unican.es
}

\section{Resumen}

Presentamos nuestro proyecto de innovación con el que pretendemos construir un espacio virtual de aprendizaje duradero complementario en la enseñanza universitaria. Tratamos de acercarnos a las metodologías activas del Aprendizaje Cooperativo y Aprendiaje Basado en Problemas como herramientas para transmitir saberes científicos.

Nuestra propuesta plantea dos ejes fundamentales para mejorar el aprendizaje: utilizar las TIC para procesar, compartir, generar y gestionar conocimientos entre profesorado y estudiantes. Además de proponer enriquecer el aprendizaje de los estudiantes con la implementación de metodologías que favorezca la adquisición de competencias.

En el curso 2011-2012, el profesorado que diseña este proyecto se ha encargado de implementar, seguir y evaluar los resultados, implicando al alumnado de diferentes asignaturas y titulaciones de la Facultad de Educación. Estudiantes que, a priori no se conocían, han 
trabajado en equipos heterogéneos resolviendo un mismo problema real, utilizando como vehículo las TIC, creando su propia red personal de aprendizaje, convertiéndola en un instrumento clave de aprendizaje y adquisición de competencias.

Presentamos algunos resultados recogidos a través de Focus Group, demostrando cómo es posible mejorar el aprendizaje universitario, en la adquisición de contenidos, el desarrollo de habilidades para trabajar en equipo en entornos virtuales y la potenciación de la metacognición.

Palabras Clave: Aprendizaje Basado en Problemas, Aprendizaje Cooperativo, TIC, Educación Superior

\section{Abstract}

Introducing our innovation project with which we intend to build a virtual learning space complementary lasting university education. We try to approach the active methodologies Cooperative Learning and Problem-Based Aprendiaje as tools to convey scientific knowledge. Our proposal raises two fundamental axes to enhance learning: using ICT to process, share, create and manage knowledge between teachers and students. Besides proposing enrich student learning by implementing methodologies that promotes the acquisition of skills. During 2011-2012, the faculty who designed this project has been responsible for implementing, monitoring and evaluating the results, involving the students in different subjects and courses at the Faculty of Education. Students who, a priori not known, heterogeneous teams have worked on solving one real problem, using ICT as a vehicle, creating their own personal learning network, converting it into a key tool for learning and skills acquisition. We present some results collected through Focus Group, demonstrating how to improve university learning in content acquisition, the development of skills to work in virtual environments and the enhancement of metacognition.

Key Words: Based Learning, Cooperative Learning, ICT, Higher Education

Fecha recepción: Febrero 2012

Fecha aceptación: Abril 2012 


\section{Introducción}

Nuestra concepción del aprendizaje, enseñanza y evaluación toma como puntos de referencia esenciales los principios que caracterizan las teorías científicas elaboradas a lo largo de la historia y que han eclosionado en las últimas décadas, anclando sus raíces en dos referentes básicos: de un lado, en la tradición evolutiva de la psicología y pedagogía europea, con una fuerte incidencia de autores, como Claparéde, Vigotsky, Bruner, Freinet, Wertsch o Feuerstein; y de otro, en la tradición experimental americana con especial incidencia de las teorías elaboradas por autores como Dewey, Ausubel, Reigeluth, Bruner, Sternberg o Freire.

Es decir, que nuestra concepción del aprendizaje toma como perspectiva los paradigmas pedagógicos que ponen el acento en el concepto clave del cambio educativo en educación superior de la década actual: la participación activa de todos los integrantes del proceso educativo. Shuell, T. J. (1986) resume la participación activa en los procesos de educación, tanto de estudiantes como de profesorado, en cinco rasgos esenciales:

- Aprendizaje activo. No es posible aprender por otra persona, sino cada persona tiene que aprender por sí misma.

- Aprendizaje autorregulado. Los estudiantes tienen que percibir sus propias actividades correctamente, evaluar los resultados de las propias actividades y retroalimentar las actividades adecuadas por sí mismos.

- Aprendizaje constructivo. El conocimiento individual no es una copia de la realidad, sino que, al menos en parte, es una construcción personal. Los estudiantes construyen su conocimiento sobre todo interpretando sus percepciones o experiencias, dependiendo de sus conocimientos u opiniones disponibles.

- Aprendizaje situado. Se comprende el aprendizaje como proceso situado cuando el contexto de aprender ofrece o, al menos, refleja oportunidades reales de aplicar los conocimientos adquiridos. 
- Aprendizaje social. Como otros procesos cognoscitivos, aprender no es un proceso exclusivamente individual, sino también un proceso social. Cada proceso de enseñanza y aprendizaje es una interacción social.

Es decir, no solo hay que tener en cuenta las cualificaciones o competencias que se están proponiendo en el nuevo marco educativo, sino los protagonistas del proceso de enseñanzaaprendizaje que han de ponerlas en juego.

Vamos a describir los enfoques teóricos relevantes según nuestra concepción de la enseñanza y el aprendizaje, elaborando breve, pero detalladamente algunas implicaciones para la enseñanza y el aprendizaje en la universidad.

A. El enfoque del constructivismo. El constructivismo es una corriente posmoderna, personalizada en Bateson, Gergen, Watzlawick, Maturana, White y otros. Uno de sus presupuestos básicos es que cuanto sabemos y creemos es fruto del lenguaje con que comprendemos y transmitimos nuestras percepciones y que, sobre una misma realidad, pueden darse diferentes puntos de vista, todos ellos igualmente válidos.

Al hablar, vamos creando la realidad junto con nuestros interlocutores. Así es como, sobre la base de nuestra biografía, creamos y modificamos nuestra identidad, retocada permanentemente en virtud del contexto y de las características y expectativas de nuestro interlocutor.

El constructivismo posmoderno considera que el cerebro no es un mero recipiente donde se depositan las informaciones, sino una entidad que construye la experiencia y el conocimiento, los ordena y da forma. Por tanto, el aprendizaje se entiende como un proceso de participación activa y adaptación del sujeto al entorno, a través del cual éste construye el conocimiento. Este principio lleva necesariamente a dos características básicas que debería tener todo aprendizaje, según García Vidal, J. (2003):

1) Significatividad, de manera que lo aprendido tenga "sentido" para el aprendiz, lo que supone sobre todo la conexión de los nuevos esquemas cognoscitivos con los que el alumno 
posee de manera previa al proceso de aprendizaje. Para el logro de aprendizajes significativos resulta básico:

- Evaluar inicialmente los conocimientos previos de los estudiantes, tanto a nivel general de la asignatura (cuestionario de evaluación inicial), como al comienzo de cada unidad de trabajo (preguntas previas, debates, diálogos y exposiciones).

- Realizar, de manera sistemática, preguntas perspicaces: durante el desarrollo de las actividades, para de esta manera, guiar a los estudiantes en el desarrollo de los procesos de construcción del conocimiento.

- Proponer ejemplos que resulten cercanos a las experiencias y conocimientos previos de los estudiantes.

- Disminuir el nivel de abstracción de los contenidos impartidos mediante la utilización de representaciones conceptuales gráfico-icónicas.

- Impulsar el empleo de mecanismos de autoevaluación, como la elaboración de estructuras conceptuales de cada unidad de trabajo.

2) Funcionalidad, principio que induce a potenciar en los aprendizajes dos características fundamentales: de un lado, el que posean la máxima relación con la vida cotidiana del estudiante; y de otro, que posean un carácter estratégico, es decir, que sirvan para adquirir nuevos aprendizajes. La consecución de aprendizajes funcionales implica:

- Relacionar los nuevos aprendizajes con experiencias y conocimientos previos de los alumnos.

- Analizar las funciones y efectos que tienen los aprendizajes realizados en la práctica profesional futura.

- Dar prioridad a los conocimientos de carácter procedimental.

B. El enfoque sociocultural. Lo fundamental del enfoque de Vygotsky consiste en considerar al individuo como el resultado del proceso histórico y social donde el lenguaje desempeña un papel esencial. Para Vygotsky, el conocimiento es un proceso de interacción entre el sujeto y el medio, pero el medio entendido social, histórica y culturalmente. Precisamente Vygotsky parte 
de que la dimensión social de la conciencia es la primaria, mientras que la dimensión individual es la secundaria y derivada de ella. Para Vygotsky "el primer problema es encontrar cómo la reacción individual procede de las formas de la vida común". Por tanto, una consecuencia importante de este pensamiento, es que para comprender los procesos cognoscitivos se tienen que entender primero los detalles de la situación social donde aparecen. Y este principio es el que ha de impulsar, sistemáticamente, en nuestra docencia, actividades como:

- Interacción continua con los estudiantes: antes de las exposiciones orales, durante y al final de las mismas. Favoreciendo que se produzcan abundantes debates sobre los contenidos que se están desarrollando en cada momento.

- Interacción continuada entre los estudiantes mediante la realización de trabajos, reflexiones y tareas sobre los conocimientos impartidos, tanto en equipo, parejas, como autónoma e individualmente.

C. El enfoque dialógico. Freire desarrolló una perspectiva dialógica en la educación en los años sesenta. Algunas veces se ha entendido esa aportación de forma restringida, limitándola al diálogo entre profesorado y alumnado dentro del aula. Pero, no es así, el diálogo propuesto por Freire abarca el conjunto de la comunidad que enseña y aprende incluyendo otros profesionales, la familia, etc., además del alumnado y del profesorado. Todos influyen en el aprendizaje y todos y todas deben planificarlo conjuntamente. El diálogo, en este contexto, se entiende como un proceso interactivo mediado por el lenguaje y que requiere, para ser considerado con naturaleza dialógica, realizarse desde una posición de horizontalidad en la que la validez de las intervenciones se encuentran en relación directa a la capacidad argumentativa de los interactuantes, y no a las posiciones de poder que éstos ocupan. Para Freire (1997) la dialogicidad es una condición indispensable para el conocimiento.

El diálogo es un instrumento para organizar este conocimiento e implica una postura crítica y una preocupación por aprehender los razonamientos que median entre los actores; y estos 
dos aspectos son los elementos que constituyen fundamentalmente la "curiosidad epistemológica" que promueve la construcción del conocimiento.

Flecha R. (1997) explica porqué todas las experiencias educativas a nivel mundial que están logrando éxitos en superación de desigualdades, se basan en las características del aprendizaje dialógico como la acción conjunta del alumnado, familias, comunidad y profesionales de la educación. Este autor cree que la importancia de este enfoque aumenta en una sociedad de la información en la que el aprendizaje depende principalmente y cada vez más de todas interacciones del alumnado y no sólo de las que recibe en el aula ni sólo de sus conocimientos previos (Flecha, R. 1999).

Por consiguiente, nuestra concepción de la enseñanza parte de la concepción del aprendizaje antes expuesta, considerando la enseñanza como una actividad al servicio de la adquisición de aprendizajes constructivos e interactivos.

Para el buen logro de esta meta consideramos esencial tener en cuenta durante el proceso de enseñanza-aprendizaje los siguientes principios (González, N., 2009):

- Enseñar a aprender. Supone el dominio de conocimientos no sólo conceptuales y actitudinales sino también procedimentales. Este principio nos lleva a dos características relevantes de nuestra actividad docente:

- Dedicar un gran esfuerzo al diseño de situaciones didácticas adecuadas a los conocimientos iniciales de los estudiantes y a las dificultades que van encontrando durante su desarrollo.

- Adaptar el nivel de complejidad de las actividades propuestas mediante la segmentación en pasos de las mismas, el trabajo cooperativo y la enseñanza de técnicas adecuadas para la resolución de problemáticas encontradas.

- Mediación. Supone una concepción docente mediadora entre los contenidos y la estructura cognitiva de los estudiantes. 
Siguiendo estas premisas optamos por aplicar las metodologías activo -participaticas del Aprendizaje Basado en Problemas y el Aprendizaje Cooperativo, conjuntamente con herramientas Web 2.0 en nuestro proyecto de innovación, que a la vez suponía un proceso de investigación-acción puesto que los estudiantes participaban y se beneficiaban conjuntamente con el profesorado de los resultados obtenidos de cara a su mejora competencial.

El aprendizaje basado en problemas es un método de enseñanza-aprendizaje que se inicia con la presentación o diseño de un problema por parte del docente, el estudiante ha de resolverlo para desarrollar determinadas competencias previamente definidas. Parte de la idea de que el estudiante apredende de un modo más adecuado cuando tiene la posibilidad de experimentar, ensayar o, sencillamente indagar sobre la naturaleza de fenómenos y actividades cotidianas. Por tanto, las situaciones problema, base del método, arrancan de situaciones de aprendizaje del mundo real.

El aprendizaje resulta mucho más estimulante cuando se plantean preguntas que requieren el esfuerzo intelectual del estudiante y no la mera repetición de una rutina de trabajo aprendida Además no se ofrece a los estudiantes toda la información necesaria para solucionar el problema, sino que son ellos los que deben identificar, encontrar, seleccionar y utilizar los recursos necesarios.

Este método se basa en la idea de que los problemas que entrañan cierta dificultad se resuelven mejor en colaboración con otras personas. Esta colaboración facilita la identificación de necesidades de aprendizaje, la búsqueda de información necesaria, la argumentación y exposición de puntos de vista o soluciones y el debate.

Actualmente se considera que el Aprendizaje Basado en Problemas puede ser uno de los métodos adecuados para los nuevos modelos de educación superior (Buck Institute for Education, 2002; Instituto Tecnológico Estudios Superiores Monterrey, 2004; Benito, A. y Cruz, A., 2005; De Miguel, M. (coord.) 2006).

El Aprendizaje cooperativo. Se trata de un enfoque interactivo de organización del trabajo fuera y dentro del aula, en el cual los estudiantes son responsables de su aprendizaje y del de sus 
compañeros, en una estrategia de corresponsabilidad para alcanzar metas e incentivos grupales.

Este método prioriza la colaboración frente a la competición. Implica una relación de compromisos y complicidades que da excelentes resultados en los ámbitos cognoscitivos y aptitudinales, pero sobre todo es un método apropiado para adquirir competencias respecto a la interacción entre iguales, la resolución de problemas y la adquisición de actitudes y valores.

Los componentes esenciales que fundamentan un aprendizaje cooperativo efectivo son: la interdependencia positiva, la interacción cara a cara, la responsabilidad individual, las habilidades sociales y la evaluación del proceso- producto (Jhonson, D, Jhonson, R. y Hulebec, E.J., 1999; Gillies, R. M. and Ashman,A.F., 2003; Monereo, C. y Duran, D., 2003).

En cuanto a las herramientas Web 2.0, hemos optado por seleccionar y proponer a nuestros estudiantes herramientas como:

- El blog articulado como un espacio de referencia en el publicar la guía del proyecto, el calendario-agenda, documentación y otros recursos online para orientar al alumnado. El alumnado, por su parte, lo utiliza para publicar sus trabajos, opiniones y otras aportaciones, de manera que queden recogidas en Internet y su acceso sea fácil y permanente, favorenciendo así el seguimiento del proyecto.

- Twitter como red de microbloging que potencia la comunicación e interacción rápida entre sus miembros mediantes mensajes cortos. Fácilmente accesible con teléfonos móviles, ampliamente generalizados en el alumnado.

- Google + como sistema de vídeo-conferencia que posibilita ir más allá de lo que ofrece Twitter al permitir la comunicación simultánea y visual entre varios participantes, independientemente del lugar donde se encuentren, además de poder compartir documentos y archivos relacionados con los trabajos durante la comunicación.

- Google Docs como aplicación orientada al trabajo colaborativo en Internet que facilita que los estudiantes trabajen tanto de manera síncrona como asíncrona, independiente del lugar desde el que lo hagan (aula de clase o desde sus casas). 


\section{DESCRIPCIÓN DEL A EXPERIENCIA}

En este proyecto pretendemos hacer realidad lo que el nuevo sistema universitario europeo promulga: darle al estudiante el protagonismo que merece, la aplicación de técnicas más acordes para el desarrollo de competencias basadas en metodologías activas y participativas, la formación en competencias y habilidades profesionales requeridas en el perfil profesionales de cada titulación, de manera que se relacione la teoría con la práctica profesional y la utilización de las TIC como instrumento de apoyo al proceso de enseñanza y aprendizaje.

En ese sentido, hemos extraido el máximo beneficio a las ventajas que aporta el trabajo en grupos heterogéneos, formados por estudiantes de diferentes asignaturas y diferentes titulaciones, de manera que cada uno ha aportado la experiencia derivada del aprendizaje propio de las asignaturas cursadas en su titulación. De esta manera, cada estudiante ha recibido la información y el conocimiento de la mano de dos responsables fundamentales: el docente y un compañero tutor del grupo. Al mismo tiempo, cada estudiante ha desarrollado la función de tutor de sus compañeros en determinadas tareas y funciones establecidas de forma previa siguiendo las pautas establecidas por expertos como Duran, d.; Torró, J. Vila, J. (2003). Por lo tanto, partimos de la concepción de que la tutoría entre iguales proporciona oportunidades de aprendizaje para todos los miembros de los equipos de trabajo.

En esta experiencia han participado tres profesores del Departamento de Educación de la universidad de Cantabria con sus respectivas asignaturas:

-- Asignatura: Didáctica de la Educación Infantil. Fundamentos teóricos. Titulación de Magisterio. Especialidad Educación Infantil, 3o curso.

-- Asignatura: Nuevas Tecnologías aplicadas a la Educación. Titulación de Magisterio. Especialidad Educación Física, 3ำ curso.

-- Asignatura: Diseño, Técnicas de Recogida de Información y Análisis de Datos Cuantitativo y Cualitativo. Titulación de Master Universitario en Investigación e Innovación en Contextos Educativos. 
El número total de estudiantes implicados en la experiencia ha sido de 9 alumnos. Se decidió seleccionar grupos de trabajo de tres estudiantes por titulación para operativizar el trabajo cooperativo en el grupo global de nueve estudiantes.

Establecimos una serie de competencias a desarrollar por todos los estudiantes implicados en la experiencia, a través de las actividades establecidas de forma conjunta, y en relación a los objetivos propuestos en el proyecto. Las competencias establecidas en el proyecto parten de unas finalidades derivadas de nuestra concepción de lo que ha de lograr la enseñanza universitaria:

1. Potenciar una mayor implicación de los estudiantes en su aprendizaje, así como en la asunción de la responsabilidad que en el mismo tienen, lo cual requiere una mayor motivación hacia las asignaturas y hacia el aprendizaje.

2. Adquirir los contenidos de la asignatura, prescindiendo de la evaluación final, y sustituyéndola por un proceso de metaevaluación continuo y reflexivo.

3. Fomentar el trabajo en equipo en entornos virtuales, a través de la práctica y de la vivencia personal

4. Dinamizar el proceso de enseñanza - aprendizaje implicándoles en su desarrollo y evaluación.

5. Desarrollar un nivel adecuado de metacognición en los estudiantes, de manera que lo aprendido en la experiencia pueda ser aplicado en su vida profesional.

La puesta en práctica supuso organizar la planificación una serie de fases, en las que concretamos las actuaciones a realizar:

A. Primera fase. Aspectos estructurales

Implementación de las herramientas digitales para desarrollar las propuestas del proyecto.

Talleres y actividades diversas de formación del profesorado y el alumnado participante (actualización de conocimientos y uso de las TICs). 
B. Segunda fase. Desarrollo del proyecto.

Seminario inicial (presencial): Destinado a la comunicación de objetivos y finalidades del proyecto, características, impacto y beneficio en la formación de los futuros docentes a todos los implicados directa e indirectamente. Se aprovecha asimismo como espacio de fomento inicial de convivencia de los agentes que toman parte en el proyecto.

Clarificar la organización del proceso de coordinación y tutorización entre las diferentes asignaturas implicadas y el fomento de la participación en grupos de trabajo cooperativo designadas.

Asimismo, se realizarán tutorías individuales y grupales (presenciales y/o virtuales).

Cada grupo recibe una consigna de actuación por parte del profesor.

A partir de este protocolo deben organizar y planificar la tarea del grupo mediante el consenso. El trabajo en los pequeños grupos se comparte y contrasta en sesiones del entorno virtual con el resto de alumnos y profesores e incluso en el propio proceso de elaboración del proyecto mediante el asesoramiento de todos.

Reuniones de coordinación quincenales (presenciales y semipresenciales) entre los profesores para establecer aspectos comunes y transversales de todas las asignaturas implicadas en el proyecto y diseñar actividades prácticas.

C. Tercera Fase. Valoración del proyecto.

Evaluar el impacto del proyecto en los agentes implicados mediante una reflexión constante contenida en las diferentes redes de aprendizaje interactivas, siendo algunos de los instrumentos utilizados: redes sociales, mensajería instantánea, seminarios presenciales y semipresenciales. 


\section{Conclusiones}

En líneas generales la valoración el profesorado participante en el diseño, implementación y desarrollo del proyecto es claramente positiva. La participación de los estudiantes les ha supuesto un incremento sustancial en su la calificaicón final de las asignaturas. La satisfacción global de los estudiantes medida con cuestionarios cerrados y el desarrollo de un grupo de discusión es realmente elevada.

Una amplia mayoría reconoce que aunque en un principio no estaban acostumbrados a organizar sus tareas de forma grupal sino individual, a lo largo del desarrollo del proyecto comenzaron a sentir los beneficios de poder "apoyarse" en sus compañeros para resolver dudas, redactar propuestas de solución y tomar decisiones antes de presentar borradores o soluciones finales a los profesores participantes, igualmente valoraron enormemente la posibilidad de contar con tres profesores y no solo uno como es habitual, para resolver dudas y problemas.

Igualmente los estudiantes valoran la potencialidad del diseño instruccional vertebrado alrededor del análisis y solución de un problema real y actual, para el desarrollo de las competencias socio-profesionales propuestas en la experiencia tales como el incremento de la motivación intrínseca, el pensamiento reflexivo, la autocrítica, la responsabilidad ante el propio aprendizaje, etc.

Creemos que el momento actual, en el que se están aplicando y poniendo a prueba las planificaciones de las nuevas titulaciones de grado en nuestras universidades, es una excelente ocasión para planificar diseños curriculares que permitan aplicar el tipo de metodologías que nosotros hemos puesto en marcha en este proyecto de innovación, en el que el profesorado se une y se coordina para diseñar un conjunto de actividades presenciales y on-line, a resolver entre estudiantes de diferentes asignaturas y especialidades, de tal modo que el estudiante, al menos en nuestra experiencia, triplica el aprovechamiento de su aprendizaje al ser supervisado y tutorizado por tres profesores y ocho compañeros más. Igualmente el profesorado reduce el número de actividades a diseñar a una única para tres asignaturas diferentes, a través de la propuesta de resolución de un problema de acuciante actualidad como el que nosotros 
propusimos titulado "Establecimiento de nuevos canales y cauces de participación para fomentar la relación familia-escuela en el ámbito rural”, en el que pudimos explicar y solicitar al estudiante el desarrollo y aplicación de los contenidos teóricos de nuestras asignaturas.

A pesar de las dificultades surgidas, que se han debido principalmente a la falta de hábito y autoplanificación de los estudiantes; a la organización de nuestros planes de estudio que promueve el desarrollo de asignaturas cuatrimestrales cargadas de actividades y trabajos que saturan la agenda de trabajo de los estudiantes, puesto que el profesorado no se coordina para proponer actividades o proyectos comunes y a la escasez de tiempo para desarrollar en profundida la resoluciónd e I problema propuesto, consideramos el resultado final satisfactorio y esperamos poder participar en la siguiente convocatoria que nuestra universidad nos propone para desarrollar la continuación de nuestro proyecto de innovación.

\section{Bibliografía}

Benito, A. \& Cruz, A. (2005). Nuevas claves para la docencia universitaria en el Espacio Europeo de Educación Superior. Madrid: Narcea.

De Miguel, M. (2006). Metodologías de enseñanza para el desarrollo de competencias. Orientaciones para el profesorado universitario ante el Espacio Europeo de Educación Superior. Madrid: Alianza.

Dirección de investigación y desarrollo educativo. Vicerrectorado Académico, Instituto Tecnológico y Estudios Superiores de Monterrey (2004). El Aprendizaje Basado en Problemas como técnica didáctica. [Documento en PDF]. Recuperado de http://www.ub.es/mercanti/abp.pdf]

Duran, D. Torró, J. Vila, J. (2003). Tutoría ente iguales. Barcelona: ICE de la UAB

Flecha, R. (1997). Compartiendo palabras. El aprendizaje de las personas adultas a través del diálogo. Barcelona: Paidós. 